\title{
Efectos de bencilaminopurina y tipo de brotes en la producción y calidad de plántulas de plátano vía macropropagación
}

Effects of benzylaminopurine and type of sprouts on the production and quality of plantain seedlings way macropropagation

Efeitos da benzilaminopurina e tipo de brotos na produção e qualidade de mudas de bananeira-da-terra através da macropropagação

\author{
Jahir López Constante ${ }^{1}$ \\ jlopez5655@utm.edu.ec \\ https://orcid.org/0000-0002-6142-0055
}

\author{
Galo Cedeño García ${ }^{2}$ \\ gcedeno@espam.edu.ec \\ https://orcid.org/0000-0002-0927-5807
}

\author{
George Cedeño García ${ }^{1}$ \\ george.cedeno@utm.edu.ec \\ https://orcid.org/0000-0001-8271-5752
}

\author{
${ }^{1}$ Universidad Técnica de Manabí, Portoviejo-Ecuador \\ ${ }^{2}$ Escuela Superior Politécnica Agropecuaria de Manabí Manuel Félix López, Calceta-Ecuador
}

Artículo recibido 3 de septiembre 2021 / Arbitrado y aceptado 8 de octubre 2021 / Publicado 13 de diciembre 2021

\section{RESUMEN}

La producción de plántulas de plátano de calidad vía macropropagación en cámara térmica es una metodología económica y eficiente para pequeños productores. El objetivo de la investigación fue evaluar el efecto de bencilaminopurina y tipo de brotes sobre la tasa de multiplicación de cormos y la calidad de plántulas de plátano propagadas en cámara térmica. Se desarrollaron dos experimentos separados. En el primero se probó el efecto de bencilaminopurina (BAP) sobre la tasa de multiplicación de cormos de plátano en cámara térmica, donde se establecieron dos tratamientos que fueron: Con BAP $\left(40 \mathrm{mg} \mathrm{L}^{-1}\right)$ y sin BAP. En el segundo se evaluó el potencial de enraizamiento, crecimiento y calidad de plántulas de plátano en vivero, generadas a partir de dos tipos de brotes en cámara térmica, que fueron: Brotes adventicios de segunda generación (BAR2) y brotes procedentes de tejido calloso (BCR2). En ambos experimentos los datos fueron analizados con prueba estadística de t de Student para observaciones pareadas. Se registró la tasa de multiplicación, variables de crecimiento y calidad de plántulas en vivero. La mayor tasa de multiplicación de brotes en cámara térmica se produjo con BAP, que supero en $44 \%$ al tratamiento sin BAP. El mayor crecimiento e índice de calidad de Dickson en plántulas durante la aclimatación fue logrado con brotes adventicios (BAR2). La calidad se correlacionó positivamente con variables morfométricas de las plántulas. El uso de BAP y brotes adventicios (BAR2) es efectivo para incrementar tasa de multiplicación y calidad de plántulas de plátano macropropagadas.

Palabras clave: Fitorregulador; Propagación; Musa AAB; Tipo de yema; Vigor de plántula
ABSTRACT

The production of quality plantain seedlings way macropropagation in a thermal chamber is an economical and efficient methodology for small producers. The aim of the research was to evaluate the effect of benzylaminopurine and type of sprouts on the multiplication rate of corms and the quality of plantain seedlings propagated in a thermal chamber. Two separate experiments were performed. 1) To test the effect of benzylaminopurine (BAP) on the multiplication rate of plantain corms in a thermal chamber, two treatments were established were: With BAP (40 $\left.\mathrm{mg} \mathrm{L}^{-1}\right)$ and without BAP. 2) To test the rooting ability, growth and seedlings quality plantain in the nursery phase generated from two types of sprouts in a thermal chamber that were: Second-generation adventitious sprouts (ASR2) and sprouts from callus tissue (CSR2). In both experiments, the data were analyzed with the Student's $t$ test for paired observations. The multiplication rate, growth variables and quality of seedlings in the nursery were recorded. The highest rate of multiplication of sprout in thermal chamber occurred with BAP, which was $44 \%$ higher than the treatment without BAP. The highest growth and quality index of Dickson in seedlings during acclimatization was achieved with adventitious sprout (ASR2). The quality was positively correlated with morphometric variables of the seedlings. The use of BAP and adventitious sprout (ASR2) is effective in increasing the multiplication rate and quality of macropropagated plantain seedlings.

Key words: Phytoregulator; Propagation; Musa $A A B$; Phytoregulator; Seedling vigour; Type of shoot

\section{RESUMO}

A produção de mudas de banana de qualidade via macropropagação em câmara térmica é uma metodologia econômica e eficiente para pequenos produtores. Oobjetivodestapesquisa foi avaliar o efeito da benzilaminopurina e do tipo de brotação na taxa de multiplicação de rizomas e na qualidade de mudas de bananeira-da-terra propagadas em câmera térmica. Dois experimentos separados foram realizados. No primeiro, testou-se o efeito da benzilaminopurina (BAP) na taxa de multiplicação de rizomas de bananeira-daterra em uma câmara térmica, onde foram estabelecidos dois tratamentos: com BAP (40 $\mathrm{mg} \mathrm{L}^{-1}$ ) e sem BAP. No segundo, avaliou-se o potencial de enraizamento, crescimento e qualidade das mudas de bananeira-da-terra em viveiro, geradas a partir de dois tipos de brotos em câmara térmica, quais sejam: brotos adventícios de segunda geração (BAR2) e brotos de tecido caloso (BCR2). Em ambos os experimentos, os dados foram analisados com o teste $t$ de Student para observações pareadas. Foram registradas a taxa de multiplicação, variáveis de crescimento e qualidade das mudas no viveiro. A maior taxa de multiplicação de brotos em câmeras térmicas ocorreu com o BAP, que foi $44 \%$ maior do que o tratamento sem BAP. O maior índice de crescimento e qualidade de Dickson em mudas durante a aclimatação foi obtido com brotos adventícios (BAR2). A qualidade está positivamente correlacionada com as variáveis morfométricas das mudas. O uso de BAP e brotos adventícios (BAR2) é eficaz para aumentar a taxa de multiplicação e a qualidade de mudas macropropagadas de bananeira-da-terra.

Palavras-chave: Fitorregulador; Propagação; Musa AAB; Tipo de broto; Vigor da muda 


\section{INTRODUCCIÓN}

El plátano (Musa AAB Simmonds) es un cultivo de gran importancia para Ecuador en el orden alimentario, social y económico, debido a que alrededor del $69 \%$ de la producción se destina al consumo local que sustenta la seguridad alimentaria del país, mientras que el restante $31 \%$ de la producción se destina a exportación, generando alrededor de 400,000 empleos, y divisas para el país $(1,2)$.

El método de propagación por regeneración natural, es el tradicionalmente usado por los productores; sin embargo, tiene como desventaja la fácil diseminación de plagas y enfermedades, lo cual disminuye significativamente la calidad sanitaria del material de siembra y por consiguiente el potencial productivo del cultivo $(3,4)$. La baja tasa de multiplicación es otra de las desventajas del método de propagación tradicional, lo cual ha sido atribuido a la dominancia apical que ejerce la planta madre sobre los retoños (5, 6). La proliferación de plántulas a través de cultivo in vitro de tejidos, es por lo general el método más adecuado de propagación, debido a las ventajas de precocidad, crecimiento homogéneo y calidad sanitaria del material vegetal obtenido. Sin embargo, este método de multiplicación involucra un alto costo de producción, por lo que los costos finales de las plántulas representan una limitante para la adquisición por parte de pequeños productores $(7,8)$.

En este contexto, la macropropagación de plátano en cámara térmica viene ganando popularidad entre los pequeños productores, debido que las altas temperaturas $\left(50-70^{\circ} \mathrm{C}\right)$ generadas estimulan la rápida brotación de yemas axilares, $y$, además, ejercen un efecto de limpieza fitosanitaria de la semilla mediante la termoterapia (9-11). En este sentido, se ha sugerido a la termoterapia como un eficaz método para la eliminación de patógenos presentes en el material de siembra, puesto que los patógenos se degradan a temperaturas inferiores al umbral térmico soportado por la semilla vegetativa $(12,13)$.

El uso de biorreguladores sintéticos como bencilaminopurina (BAP), benciladenina (BA) y thidiazuron (TZA) han mostrado potencial para incrementar la tasa de multiplicación del banano vía micropropagación y macropropagación $(10,14,15)$. En este sentido, resultados obtenidos por Thiemele et al. (16) y Cedeño et al. (10) concluyeron que la aplicación de BAP en dosis de $40 \mathrm{mg} \mathrm{L}^{-1}$, incrementaron la producción de plántulas de banano en un 50 y $80 \%$, respectivamente, en relación a los tratamientos controles. Resultados similares fueron encontrados por Ramirez et al. (17) y Opata et al. (15) con el uso de BAP en la macropropagación de plántulas de banano en cámaras de crecimiento.

Por otra parte, se ha sugerido que el tipo y tamaño de explante o yemas obtenidos en los procesos de macropropagación, es determinante para potenciar el enraizamiento, sobrevivencia, crecimiento temprano y calidad de las plántulas de banano en fase de vivero (18-20).

Los principales resultados de investigaciones previas han enfocado sus 
estudios de macropropagación principalmente en banano, por lo que existe escasa información relacionada a macropropagación del plátano; lo cual sumado a una creciente demanda de tecnologías de propagación asequible y de bajo costo por pequeños productores, que no pueden acceder a material de siembra de calidad proveniente de cultivo de tejido por limitantes económicas, son justificativos de la presente investigación. Por lo anterior, el objetivo principal del trabajo fue evaluar el efecto de bencilaminopurina y tipo de brotes sobre la tasa de multiplicación de cormos y la calidad de plántulas de plátano propagadas en cámara térmica.

\section{MATERIALES Y MÉTODOS}

\section{Localización de la investigación}

La investigación se desarrolló durante la época seca del año 2019, en el campus experimental de la Escuela Superior Politécnica Agropecuaria de Manabí Manuel Félix López, en Calceta, Manabí, Ecuador. El ensayo se ubicó geográficamente en las coordenadas $0^{\circ} 49^{\prime} 10^{\prime \prime}$ de latitud sur y $80^{\circ} 10^{\prime} 40^{\prime \prime}$ de longitud oeste, a una altitud de $21 \mathrm{msnm}$.

Se desarrollaron dos experimentos separados como se indica a continuación:

Experimento 1. Efecto de bencilaminopurina (BAP) sobre la tasa de multiplicación de cormos de plátano en cámara térmica.

\section{Tratamientos, diseño estadístico y análisis de datos}

Se evaluaron dos tratamientos que fueron. Propagación con BAP y propagación sin BAP. Cada uno de los tratamientos se conformó por 10 repeticiones de $1 \mathrm{~m}^{2}$ y un total de 20 unidades experimentales. La unidad experimental fue conformada por $12 \mathrm{cormos} / \mathrm{m}^{2}$. Para comparar el efecto entre tratamientos se utilizó la distribución de "t de Student" para muestras pareadas, donde se probaron la hipótesis nula $\mathrm{H}_{0}: \mathrm{T}_{1}=\mathrm{T}_{2}$ y alternativa $\mathrm{H}_{1}: \mathrm{T}_{1} \neq \mathrm{T}_{2^{\prime}}$ a un nivel de significancia del $5 \%$ de probabilidades de error. El cálculo del estadístico de t se realizó mediante la fórmula siguiente:

$$
t_{c a l}=\frac{\bar{d}}{S_{\bar{d}}}
$$

Donde $t=$ estadístico; $\vec{a}=$ media de las diferencias; $S_{\bar{d}}=$ error estándar de las diferencias.

\section{Material vegetal}

Para el experimento se utilizaron cormos de plátano cv. Dominico-Hartón de $4 \mathrm{~kg}$ de peso, procedentes de hijos espada. Los cormos fueron limpiados con un cuchillo removiendo partes necrosadas hasta dejarlos completamente blancos con las yemas axilares expuestas; esto con el interés de remover partes biológicas de artrópodos plaga, nematodos y agentes 
patógenos. Seguidamente, fueron sometidos a termoterapia mediante la sumersión en agua hirviendo por 25 segundos, con el fin de garantizar su estado sanitario de acuerdo a lo expuesto por Coyne et al. (21). Luego, se procedió a extirpar el punto de crecimiento del cormo madre con la ayuda de un cuchillo previamente desinfectado, con la finalidad de inhibir la dominancia apical ejercida por las auxinas, promover la activación, crecimiento y formación de multiyemas y tejido calloso.

\section{Aplicación de BAP}

Una vez desinfectados los cormos e inhibida su dominancia apical, se procedió a la aplicación de una solución de $4 \mathrm{~mL}$ de BAP a una concentración de $40 \mathrm{mg} \mathrm{L}^{-1}$. La solución de BAP fue aplicada en la cavidad dejada en el centro del cormo por la extirpación del punto de crecimiento.

Luego de este procedimiento, los cormos fueron dejados por 24 horas a la sombra con la finalidad de permitir que la solución de BAP aplicada sea absorbida por el tejido del cormo y evitar que su descomposición por radiación solar. Finalmente, pasado este periodo, los cormos fueron introducidos y sembrados en cámara térmica.

\section{Cámara térmica}

La cámara fue construida a base de caña guadua y madera, con tamaño de $2 \times 10 \times 1,5 \mathrm{~m}$ de ancho, longitud y altura, respectivamente. La estructura fue cubierta con plástico transparente de $0,8 \mathrm{~mm}$ de espesor y anti UV, además de malla saran al $50 \%$, con la finalidad de evitar la quemadura de las plántulas. En el interior de la cámara térmica se construyeron platabandas sobre el nivel de suelo, de 1,0 $\mathrm{m}$ de ancho y $0,25 \mathrm{~m}$ de altura, que fueron llenadas con sustrato compuesto por suelo de capa arable, compost y cascarilla de arroz en proporción 2:1:1. Seguidamente, los cormos fueron colocados semienterrados en el sustrato de las platabandas. Una vez iniciada la emisión de brotes de primera generación (R1), se procedió a inhibir la dominancia apical de estos brotes, con la ayuda de una navaja punta plana desinfectada, con la cual se extirpó el punto de crecimiento en forma de asterisco, con el fin de estimular la formación de yemas axilares (organogénesis directa) y masa de tejido calloso (organogénesis indirecta) para finalmente obtener plántulas de segunda generación (R2) (Figura 1A, 1B, 1C). Independientemente de los niveles de BAP los cormos recibieron una fertilización con fertilizante soluble compuesto por $12 \%$ de $\mathrm{N}$, $19 \%$ de $\mathrm{P}_{2} \mathrm{O}_{5}, 18 \%$ de $\mathrm{K}_{2} \mathrm{O}, 2,7 \%$ de $\mathrm{MgO}, 20 \%$ de $\mathrm{SO}_{4}{ }^{-2}, 0,02 \%$ de $\mathrm{Mn}, 0,015 \%$ de $\mathrm{B}, 0,2 \%$ de $\mathrm{Fe} y$ $0,02 \%$ de $\mathrm{Zn}$, en dosis de 10 gramos por cormo.

\section{Variables respuesta}

Las variables registradas para el análisis de efectos de los tratamientos fueron: Tasa de multiplicación de cormos (TM) que se registró al final del experimento cuantificando el total de plántulas producidas por cormo a los 90 días después de iniciada la emergencia de los brotes R1, de acuerdo a la ecuación [1]. La producción del número de plantas por $\mathrm{m}^{2}$ fue registrado contabilizando el número de plantas totales cosechadas por $\mathrm{m}^{2}$ a los 90 días después de iniciada la emergencia de los 
brotes R1.

\section{Plantas totales por cormo \\ $T M=$ Número de cormos iniciales [1]}

Experimento 2. Potencial de enraizamiento, crecimiento y calidad de plántulas de plátano en vivero, generadas a partir de dos tipos de brotes en cámara térmica

\section{Tratamientos, diseño estadístico y análisis de datos}

Se evaluaron dos tipos de brotes obtenidos en cámara térmica que fueron: brotes adventicios de segunda generación (BAR2) formados a partir de yemas axilares del brote R1 y brotes de segunda generación formados a partir de tejido calloso (BCR2) del brote R1, los cuales se muestran en la figura 1B y 1C (Figura 1). Cada uno de los tratamientos se conformó por 10 repeticiones y un total de 20 unidades experimentales. La unidad experimental fue conformada por 20 plántulas. Para comparar el efecto entre tratamientos se utilizó la distribución de "t de Student" para muestras pareadas, donde se probaron la hipótesis nula $H_{0}: T_{1}=T_{2}$ y alternativa $H_{1}: T_{1} \neq T_{2}$, a un nivel de significancia del $5 \%$ de probabilidades de error. El cálculo del estadístico de t se realizó mediante la fórmula siguiente:

$$
t_{c a l}=\frac{\bar{d}}{S_{\bar{d}}}
$$

Donde $t=$ estadístico; $\vec{a}=$ media de las diferencias; $S_{\bar{d}}=$ error estándar de las diferencias.

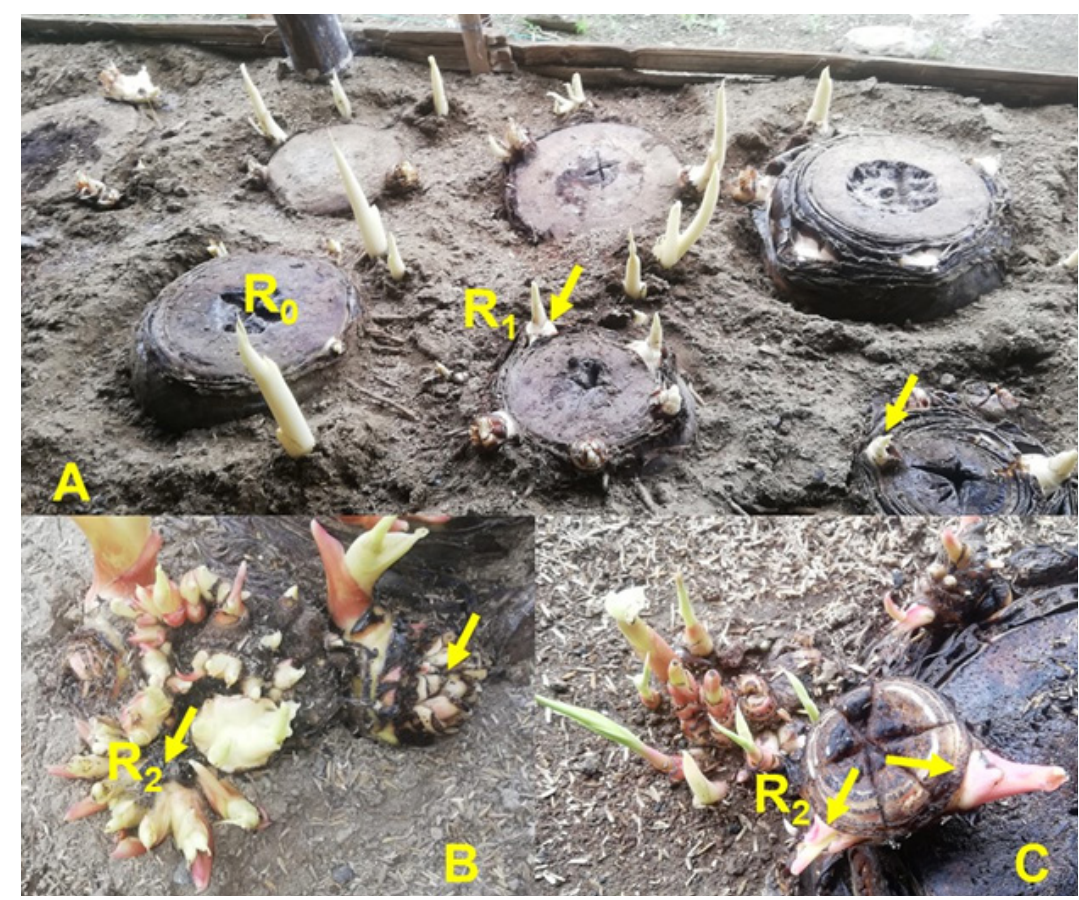

Figura 1. Brotes de primera generación R1 formados a partir del cormo madre R0 (A). Brotes R2 formados a partir de tejido calloso del R1 (B). Brotes adventicios R2 formados a partir de yemas axilares del R1 (C). 


\section{Manejo del material vegetal}

Para el experimento se extrajeron desde la cámara térmica brotes R2 adventicios (BAR2) y procedentes de tejido calloso (BCR2), que presentaron al menos la hoja bandera. Seguidamente, la base de los brotes fue sumergida en agua destilada estéril durante 15 días, con la finalidad de estimular el enraizamiento según la metodología propuesta por Cedeño et al. (19). Los brotes que después de este tiempo no emitieron raíces, se consideraron como muertos. Luego del enraizamiento, los brotes fueron sembrados en contenedores de polietileno de $6 \times 9$ pulgadas de dimensión, que fueron llenadas previamente con sustrato a base de suelo de capa arable, arena de río y vermicompost, en proporción 2:1:1. A partir del trasplante a contenedores, las plántulas se dejaron crecer por un periodo de 60 días en vivero cubierto con malla sarán al $50 \%$ de sombra.

\section{Variables respuesta}

A los 15 días después de sumergir la base de los brotes en agua para estimular emergencia de raíces, se registró el porcentaje de enraizamiento (\%), longitud de masa radical (cm) y masa seca radical (g). A los 60 días de aclimatación en vivero, fue registrada la altura de planta $(\mathrm{cm})$, el diámetro del tallo $(\mathrm{mm})$, la longitud de biomasa radical $(\mathrm{cm})$, la masa seca de planta $(\mathrm{g})$, área foliar $\left(\mathrm{cm}^{2}\right)$ e índice de calidad de Dickson. El porcentaje de enraizamiento en agua fue medido con la ecuación [2].
$P E(\%)=\frac{\text { Número de brotes enraizados }}{\text { Número de brotes totales }} \times 100$

La longitud de masa radical tanto a los 15 días de enraizamiento en agua fue registrada desde la base del rizoma hasta la zona apical del conjunto de raíces principales. La masa seca radical tanto a los 15 días de enraizamiento en agua y la masa seca de planta a los 60 DDT, fue determinada con la ayuda de una estufa de ventilación forzada a $75^{\circ} \mathrm{C}$ hasta alcanzar peso constante. La altura de planta fue registrada a los 60 DDT, para lo cual se midió desde el nivel del suelo hasta la $V$ formada por la intersección de las dos últimas hojas. El diámetro del tallo se registró a los 60 DDT, lo cual se realizó a nivel del suelo con la ayuda de un calibrador. El área foliar fue determinada a los 60 DDT con la metodología de la ecuación [3].

Área Foliar $\left(\mathrm{cm}^{2}\right)=L H * A H * K(0.80) * N H * K_{2}(0.662)$ [3]

Dónde: $\mathrm{LH}=$ longitud de tercera hoja, $\mathrm{AH}=$ ancho de tercera hoja, $\mathrm{K}$ = factor de curvatura de Murray (22), $\mathrm{NH}=$ número de hojas y $\mathrm{K}_{2}=$ nuevo factor de curvatura de Kumar et al. (23).

Finalmente, el índice de calidad de Dickson (ICD) fue determinado a los 60 DDT con la metodología de la ecuación [4], la cual integra todas las variables antes mencionadas.

$$
I C D=\frac{\text { Masa seca de planta }(\mathrm{g})}{\frac{\text { Altura de planta }(\mathrm{cm})}{\text { Diámetro de pseudotallo }(\mathrm{mm})}+\frac{\text { Masa seca aerea }(\mathrm{g})}{\text { Masa seca radical }(\mathrm{g})}}
$$




\section{RESULTADOS Y DISCUSIÓN}

Experimento 1. Efecto de bencilaminopurina (BAP) sobre la tasa de multiplicación de cormos de plátano en cámara térmica.

La aplicación de BAP influyó significativamente $(p<0,05)$ la tasa de multiplicación de cormos y la cantidad de plántulas producidas por $\mathrm{m}^{2}$ de cámara térmica, lo que podría sugerir que el fitorregulador estimula procesos de división, crecimiento y diferenciación celular a nivel de yemas vegetativas preformadas en el rizoma. El efecto de BAP incrementó la producción de plántulas en un $44 \%$ en relación al tratamiento sin BAP, tanto a nivel de rizoma como por superficie de cámara térmica (Tabal 1). Los resultados hallados, guardan similitud con los encontrados por Thiemele et al. (16) quienes reportaron en promedio una producción de
280 plántulas con aplicación de $40 \mathrm{mg} \mathrm{L}^{-1}$ de BAP, en relación a las 165 obtenidas sin BAP. Del mismo modo, Cedeño et al. (10) reportaron una tasa de multiplicación de 47 plántulas en cormos de banano $\mathrm{cv}$. Williams tratados con 40 $\mathrm{mg} \mathrm{L}^{-1}$ de BAP, en contraste a las 26 plántulas obtenidas en cormos no tratados con BAP. En este mismo contexto, resultados cercanos fueron hallados por Ramírez et al. (17) quienes alcanzaron tasas de multiplicación en cormos de banano tratados con BAP de hasta 73 , en relación a las 27 plántulas producidas por el tratamiento control sin BAP. Así mismo, los resultados hallados coinciden a los reportados por Opata et al. (15) quienes lograron tasa de multiplicación de hasta 33 plántulas en cormos tratados con BAP, en comparación a las 24 plantas producidas en cormos no tratados con BAP.

Tabla 1. Tasa de multiplicación del plátano propagado en cámara térmica en función de BAP.

\begin{tabular}{|c|c|c|c|c|c|c|}
\hline \multirow{2}{*}{ Variables } & \multicolumn{2}{|c|}{$\begin{array}{l}\text { Medias tratamientos en } \\
\text { función de BAP }\end{array}$} & \multirow{2}{*}{$\bar{d}$} & \multirow{2}{*}{$\mathbf{T}_{\text {estadistico }}$} & \multirow{2}{*}{$\begin{array}{l}\text { Valor crítico } \\
\quad \text { de } t_{0.05}\end{array}$} & \multirow{2}{*}{ p-valor } \\
\hline & $\begin{array}{l}\text { Con } \\
\text { BAP }\end{array}$ & $\begin{array}{l}\text { Sin } \\
\text { BAP }\end{array}$ & & & & \\
\hline Tasa de multiplicación de cormos & 32 & 18 & 14,00 & 4,82 & 2,23 & 0,0005 \\
\hline Número de plántulas por m2 & 378 & 213 & 165,00 & 4,82 & 2,23 & 0,0005 \\
\hline
\end{tabular}


El incremento de la tasa de multiplicación en cormos tratados con BAP, puede deberse a una mayor concentración de citocininas alcanzada en los rizomas con relación a las auxinas, lo cual favoreció el balance hormonal a favor de las citocininas y por ende mayor estimulación de meristemos axilares. Esta teoría fue propuesta por Skoog y Miller en 1957 y actualmente sigue siendo aceptada por la comunidad científica (24-26). En este sentido, se ha demostrado que la aplicación de citocininas exógenas a algunos órganos que normalmente carecen de esta hormona induce la división celular y su efecto se ha relacionado con todas las etapas del ciclo celular. Además, se ha informado que la dominancia apical se suprime bajo el efecto de una alta concentración de citoquininas y permite el desarrollo de yemas axilares (25, $27,28)$.
Experimento 2. Potencial de enraizamiento, crecimiento y calidad de plántulas de plátano en vivero, generadas a partir de dos tipos de brotes en cámara térmica.

Durante el enraizamiento en agua, el tipo de plántula influyó significativamente $(p<0.05)$ sobre el enraizamiento y crecimiento radical de las plántulas (Tabla 2). El porcentaje de enraizamiento en agua fue $9.10 \%$ superior en plántulas adventicias, con relación a las plántulas formadas a partir de callos. Del mismo modo, las plántulas adventicias mostraron una longitud radical $16.69 \%$ superior al de las plántulas provenientes de callo. Así mimo, la masa seca radical fue un 32.63 \% superior en plántulas adventicias, en relación a las provenientes de callos (Tabla 2).

Tabla 2. Potencial de enraizamiento y crecimiento radical de dos tipos de brotes $\mathrm{R} 2$ obtenidas en cámara térmica a los 15 días después de sumersión en agua.

\begin{tabular}{lcccccc}
\hline \multicolumn{1}{c}{ Variables } & $\begin{array}{c}\text { Medias tratamientos en función } \\
\text { del tipo de brote }\end{array}$ & $\boldsymbol{d}$ & $\mathbf{T}_{\text {estadistico }}$ & $\begin{array}{c}\text { Valor crítico } \\
\text { de }_{0.05}\end{array}$ & p-valor \\
\hline Brote adventicio & Brote de callo & & & & \\
\hline Enraizamiento (\%) & 82,01 & 74,55 & 7,46 & 2,58 & 2,10 & 0,0423 \\
Longitud radical (cm) & 6,53 & 5,44 & 1,09 & 2,86 & 2,72 & 0,0354 \\
Masa seca radical $(\mathrm{g})$ & 1,90 & 1,28 & 0,63 & 2,72 & 2,45 & 0,0235 \\
\hline
\end{tabular}

En cuanto a la respuesta de enraizamiento en agua de los dos tipos de plántulas evaluadas, los resultados contradicen a los hallados por Cedeño et al. (19), quienes reportaron mayor porcentaje de enraizamiento en plántulas de banano cv. William procedentes de callos con $86.4 \%$, en relación a plántulas adventicias con $79.5 \%$. Lo anterior, puede estar en función del genotipo, dado que el banano cv. William pertenece al grupo genómico AAA, mientras 
que el plátano al grupo genómico $A A B$. En este contexto, resultados obtenidos por Reyes et al. (29) reportaron que el plátano cv. Barraganete (AAB) mostro menores tasas de supervivencia y regeneración, en relación al cultivar de banano William (AAA).

La mayor respuesta de enraizamiento y crecimiento radical en plántulas adventicias de plátano con relación a las procedentes de masas de callo, puede deberse al mayor tamaño de las plántulas adventicias, puesto que estas crecen de forma aislada e independiente y por tanto pueden acumular mayores reservas nutricionales en sus tejidos y por ende mayor crecimiento $(30,31)$, en relación a las plántulas procedentes de tejido calloso que crecen agrupadas y con mayor competencia por recursos nutricionales (Figura 1). Independientemente del tipo de plántula, el enraizamiento en agua se presenta como una alternativa adecuada para la estimulación de raíces en explantes de plátano obtenidos a partir de rizomas sometidos a propagación en cámara térmica. En este contexto, resultados obtenidos por Dawood et al. (32), reportaron que el agua estimuló la formación de raíces en Solanum dulcamara a partir de meristemos preexistentes, que se debió a la activación de genes relacionados a la hipoxia y etileno. Por otra parte, se ha sugerido que la inducción y desarrollo de raíces en plántulas sumergidas en agua, está regulada por una red de señalización que promueve la síntesis de auxinas y etileno, mientras que por el contrario la producción de citocininas y estrigolactonas se ve inhibida (33).
A los 60 días de aclimatación en vivero, el tipo de planta influenció significativamente $(p<0.05)$ todas las variables de crecimiento y la calidad de las plántulas (Tabla 3). La altura, diámetro de tallo y longitud radical de las plántulas adventicias fueron un 34.48, 25.22 y $18.82 \%$ superior en su orden respectivo, a las plántulas procedentes de tejido calloso. Así mismo, la acumulación de masa seca de plántulas adventicias se incrementó en un $38.26 \%$ en comparación a las plántulas de callo. Del mismo modo, el área foliar de la plántula adventicia superó al de las plántulas procedentes de tejido calloso con un $34.56 \%$. Finalmente, las plántulas adventicias también mostraron mayor índice de calidad de Dickson con un 22.89 \% en relación a las plántulas obtenidas de callo. Los resultados obtenidos indican claramente que las plántulas adventicias muestran un mayor crecimiento y calidad a los 60 días de aclimatación en vivero (Tabla 3). El mayor crecimiento y calidad logrado por las plántulas adventicias puede deberse a un mayor tamaño y vigor inicial en comparación con las procedentes de callo (Figura 1). En este contexto, varios estudios han sugerido que explantes y semillas de mayor tamaño en otras especies de plantas, dependen en menor medida de los nutrientes obtenidos del medio donde se desarrollan u originan, debido a la mayor concentración de reservas nutrimentales acumuladas en sus órganos y tejidos, que les proporciona mayores recursos metabólicos para la formación de nuevas estructuras y el crecimiento inicial (31, $34,35)$. 
Tabla 3. Crecimiento y calidad de plántulas de plátano en vivero obtenidas a partir de dos tipos de brotes en cámara térmica a los 60 días de aclimatación en vivero.

\begin{tabular}{lcccccc}
\hline \multicolumn{1}{c}{ Variables } & $\begin{array}{c}\text { Medias tratamientos en función } \\
\text { del tipo de brote }\end{array}$ & $\boldsymbol{d}$ & $\mathbf{T}_{\text {estadistico }}$ & $\begin{array}{c}\text { Valor crítico } \\
\text { de t }_{0.05}\end{array}$ & p-valor \\
& Brote adventicio & Brote de callo & & & & \\
\hline Altura de planta (cm) & 39,68 & 26,00 & 13,68 & 6,62 & 3,55 & 0,0217 \\
Diámetro de tallo (mm) & 32,00 & 23,93 & 8,17 & 11,48 & 9,57 & 0,0377 \\
Longitud radical (cm) & 66,67 & 54,12 & 12,55 & 11,49 & 10,68 & 0,0393 \\
Masa seca de planta (g) & 37,90 & 23,40 & 14,50 & 8,53 & 2,96 & 0,0135 \\
Área foliar (cm ${ }^{2}$ ) & 4415,91 & 2889,75 & 1526,16 & 6,31 & 3,00 & 0,0242 \\
$\begin{array}{l}\text { Índice de calidad de } \\
\text { Dickson (ICD) }\end{array}$ & 11,36 & 8,76 & 2,60 & 3,88 & 3,00 & 0,0235 \\
\hline
\end{tabular}

El hecho de que plántulas provenientes de explantes o brotes de mayor tamaño presenten mayores tasas de crecimiento durante la aclimatación en vivero y campo, ha sido confirmado por varios estudios. En este sentido, Ashango (36) obtuvo mayor altura, diámetro y hojas de botes obtenidos a partir de cormos de Ensete ventricosum de mayor tamaño, en comparación a los de menor dimensión. Por su parte, Patiño et al. (37) informaron que rizomas de banano bocadillo entre 300 - 700 y 700 - 1000 g produjeron plántulas con mayor altura, grosor de tallo y número de hojas, en contraste a rizomas de menor tamaño. En este mismo contexto, Rai et al. (20) reportaron mayor altura, diámetro de tallo, longitud radical, masa seca y hojas en plántulas de banano obtenidas de rizomas de $9 \times 9 \times 9 \mathrm{~cm}$, en contraste a rizomas de menor volumen.
Finalmente, resultados logrados por Muhidin et al. (38) reportaron mayor altura de planta, circunferencia de tallo y cantidad de hojas a los 70 días después de trasplante, en rizomas de banano cv. Dwarf de $12 \times 12 \times 12$ $\mathrm{cm}$, en contraste a plántulas procedentes de rizomas de menor dimensión. En lo relacionado al índice de calidad de Dickson, los resultados obtenidos fueron cercanos a los alcanzados por Rodríguez y Ramírez (39) en plántulas de banano en fase de vivero, con promedios de hasta 12 de ICD.

Independientemente del tipo de plántulas evaluada, el crecimiento y la calidad se correlacionaron positiva y significativamente $(p<0.05)$ a los 60 días en etapa de vivero (Tabla 4). 
Tabla 4. Relación entre el crecimiento y calidad de plántulas de plátano propagadas en cámara térmica a los 60 días de aclimatación en vivero.

\begin{tabular}{llllll}
\hline & \multicolumn{1}{c}{$\begin{array}{c}\text { Altura de } \\
\text { planta }(\mathbf{c m})\end{array}$} & $\begin{array}{c}\text { Diámetro de } \\
\text { tallo }(\mathbf{m m})\end{array}$ & $\begin{array}{c}\text { Longitud } \\
\text { radical }(\mathbf{c m})\end{array}$ & $\begin{array}{c}\text { Masa seca de } \\
\text { planta }(\mathbf{g})\end{array}$ & $\begin{array}{c}\text { Área foliar } \\
(\mathbf{c m})\end{array}$ \\
\hline & & & & & \\
Índice de calidad de Dickson & $R=0.85$ & $R=0.80$ & $R=0.85$ & $R=0.86$ & $R=0.82$ \\
$(I C D)$ & $P=0.0342$ & $P=0.0450$ & $P=0.0312$ & $P=0.0284$ & $P=0.0473$ \\
\hline
\end{tabular}

$\mathrm{R}=$ Coeficiente de correlación de Pearson

$\mathrm{P}=$ significancia estadística de las correlaciones $\quad \leq 0.05$

En este sentido, reportes hechos por Binotto et al. (40), concluyeron que la masa seca y el diámetro del tallo son las variables que expresan mayor correlación con el índice de calidad de Dickson. Por su parte estudios realizados por Lin et al. (41) concluyeron que variables morfofisiológicas se correlacionan con el vigor de las plántulas. Finalmente, se ha demostrado que la calidad de las plántulas está relacionada con mejores índices morfofisiológicos, que conlleva a mayor potencial de crecimiento y capacidad de tolerar el estrés postrasplante a campo definitivo $(41,42)$.

\section{CONCLUSIONES}

El uso de bencilaminopurina (BAP) en dosis de $40 \mathrm{mg} \mathrm{L}^{-1}$, fue efectivo para incrementar la tasa de multiplicación del plátano en cámara térmica. Las plántulas provenientes de brotes adventicios de segunda generación mostraron mayor enraizamiento y crecimiento en fase de vivero, con relación a las plántulas formadas a partir de tejido calloso. Las plántulas obtenidas a partir de brotes adventicios lograron mayor calidad en vivero. La calidad de las plántulas en vivero se correlacionó con mejores caracteres morfométricos.

\section{Agradecimientos}

Los autores agradecemos a la Escuela Superior Politécnica Agropecuaria de Manabí Manuel Félix López, por haber financiado parte de esta investigación a través del proyecto institucional CUP 381232 "Selección de clones élites de plátano (Musa AAB Simmonds.) de alto potencial productivo a partir de fincas plataneras de Manabí", que se ejecutó durante el periodo 2017-2021 como consta en la Secretaría de Planificación del Estado Ecuatoriano.

\section{REFERENCIAS BIBLIOGRÁFICAS}

1. Beltrón C, Sánchez A, Ortiz M. El fortalecimiento de la comercialización del plátano mediante formas asociativas. caso de estudio del cantón El Carmen de la provincia de Manabí. Revista Caribeña de Ciencias Sociales. 2018. https://n9.cl/198oc (consulta de oct. 12, 2021)

2. MAG (Ministerio de Agricultura y Ganadería). Sistema de Información Pública Agropecuaria, Ficha técnica del cultivo de plátano (Musa AAB). 2020. https://n9.cl/5d6fp (consulta de Dic.15, 2020) 
3. Njau N, MwangiM, GathuR, Mbaka J, Muasya R. Banana weevil (Cosmopolites sordidus) reduces availability of corms for seedling production through macropropagation technology. Journal of Animal \& Plant Sciences 2011;12(1):1537-1542

4. Jacobsen $\mathrm{K}$, Omondi $\mathrm{B}$, Almekinder $\mathrm{C}$, Alvarez, E, Blomme G, Dita M, Iskra M, Ocimati W, Tinzaara, W, Kumar P, Staver C. Seed degeneration of banana planting materials: strategies for improved farmer access to healthy seed. Plant Pathology 2019;68(1):207228

5. Bangata $B$, Mobambo $K$, Kasongo $M$, Shungu D, Vuvu K, Vangu P, Omondi A, Staver C. Évaluation du potentiel prolifératif de six cultivars de bananier (cv. AAB, ABB, et AAA) par macropropagation en République Démocratique du Congo. Journal of Applied Biosciences 2018;127(1):12770-12784

6. Baganta B, Ngbenelo N, Mobambo K. Evaluation du potentiel de prolifération d'explants de différentes dimensions de bananier plantain (Musa sp. cv. AAB) para la macropropagation en conditions semicontrôlées. Revue Africaine d'Environnement et d'Agriculture 2019;2(2): 25-31

7. Mugo S, Bunde A, Korir M, Mundaki J. Factors Influencing Tissue Culture Banana Output and its Impact on Income in Nyamusi Division, Nyamira North District, Kenya. International Journal of Sciences: Basic and Applied Research (IJSBAR) 2013;2: 1-24

8. Tumuhimbise R, Talengera D. Improved propagation techniques to enhance the productivity of banana (Musa spp.). Open Agriculture 2019; 3:138-145

9. Álvarez E, Ceballos G, Gañán L, Rodríguez D, González S, Pantoja A. Producción de material de siembra limpio en el manejo de las enfermedades limitantes del plátano. Centro Internacional de Agricultura Tropical (CIAT), 2013, Cali, CO. 16 p

10. Cedeño G, Soplín H, Helfgott S, Cedeño G, Sotomayor I. Aplicación de biorreguladores para la macro- propagación del banano cv. Williams en cámara térmica. Agronomía Mesoamericana 2016;27(2): 397-408

11. Ntamwira J, Sivirihauma C, Ocimati W, Bumba M, Vutseme L, Kamira M, Blomme G. Macropropagation of banana/plantain using selected local materials: A cost-effective way of mass propagation of planting materials for resource-poor households. European Journal of Horticultural Science 2017;82(1):38-53

12. Lassois $L$, Lepoivre $P$, Swennen $R$, van den Houwe I, Panis B. Thermotherapy, Chemotherapy, and Meristem Culture in Banana. In: Humana Press, Totowa (ed.). 2013, New Jersey. pp. 419-433

13. Panattoni A, Luvisi A, Triolo E. Review. Elimination of viruses in plants: twenty years of progress. Spanish Journal of Agricultural Research 2013;11(1):173-188

14. Deo B, Keshari B, Pradhan B. In vitro propagation of popular banana cultivar (Musa spp. Cv. Patakpura). Bangladesh Journal of Agricultural Research 2019;44(4):641-648

15. Opata J, Melichar $P$, Hegele M, Hegele S, Dzomeku B, Wünsche J. Macropropagation of plantain (Musa $A A B$ ): Responses to hormonal and mechanical corm manipulation. Fruits 2020;75(3):123-129

16. Thiemele D, Issali A, Traore S, Kouassi K, Aby N, Gnonhouri P, Kobenan J, Yao T, Adiko A, Zakra A. Macropropagation of plantain (Musa spp.) Cultivars PITA 3, FHIA 21, ORISHELE and CORNE 1: effect of benzylaminopurine (BAP) concentration. Journal of Plant Development 2015; 22:31-39

17. Ramirez J, Batoom G, Sacayanan A. Macropropagation of Saba Banana Using Misting System and Different Plant Growth Enhancers. MAYFEB Journal of Agricultural Science 2016; 4:28-33

18. Sosa F, Ortiz R, Hernández R, Armas $O$, Guillen D. Propagación in vitro de Heliconia standley Macbride en Cuba. Revista Chapingo Serie Horticultura 2009;15(2):17-23 
19. Cedeño G, Soplín H, Cargua J, Cedeño G. Potencial de enraizamiento en agua y vigor de plántulas de banano obtenidas en cámara térmica. La Técnica Revista de Las Agrociencias 2016;16(1):6-15

20. Rai I, Sudana I, Gede I, Dwiyani R. The Effect of Chopped Corm Sizes and Types of Soaking Material on Growth Seedling of Kepok Banana (Musa paradisiaca L. ABB Kepok). International Journal of Life Sciences 2020;4(2):32-41

21. Coyne D, Wasukira A, Dusabe J, Rotifa I, Dubois T. Boiling water treatment: A simple, rapid and effective technique for nematode and banana weevil management in banana and plantain (Musa spp.) planting material. Crop Protection 2010;29(12):1478-1482

22. Murray D. The effect of deficiencies of the major nutrients on growth and leaf analysis of the banana. Tropical Agriculture 1960;37(2):97106

23. Kumar N, Krishnamoorthy V, Nalia L, Soorianathasundharam K. A new factor for estimating total leaf area in banana. InfoMusa 2002;11(2):42-43

24. Cheng $Z$, Wang $L$, Sun $W$, Zhang $Y$, Zhou $C$, Su Y, Li W, Sun T, Zhao X, Li X, Cheng Y, Zhao Y, Xie Q, Zhang X. Pattern of Auxin and Cytokinin Responses for Shoot Meristem Induction Results from the Regulation of Cytokinin Biosynthesis by AUXIN RESPONSE FACTOR3. Plant Physiology 2013;161(1):240-251

25. Kieber J, Schaller G. Cytokinins. The Arabidopsis Book/American Society of Plant Biologists 2014;12: 168

26. Su $Y$, Zhang $X$. The Hormonal Control of Regeneration in Plants. Current Topics in Developmental Biology 2014; 108:35-69

27. Pereira G, Santaella M, Alves L, Silva E, Flenga A, Santos D. Concentrations of 6-Benzylaminopurine (BAP) in micropropagation of banana 'Farta Velhaco' (AAB). Comunicata Scientiae 2018;9(1):58-63

28. Shin J, Bae S, Seo P. De novo shoot organogenesis during plant regeneration. Review paper. Journal of Experimental Botany 2020;71(1):63-72
29. Reyes G, García J, Piña F, Mendoza J, Sosa D, Noceda C, Blasco M, Flores J. In Vitro Proliferation and Cryoconservation of Banana and Plantain Elite Clones. Journal of Horticultural Research 2017;25(2):37-47

30. Taji A, Williams R. Use of in vitro breeding strategies in the development of Australian native plants. Acta Horticulturae 2005; 683:8793

31. Smith R. Plant Tissue Culture (Third Edition). Chapter 4 - Explant Preparation. Academic Press Elsevier 2013. San Diego

32. Dawood T, Rieu I, Wolters-Arts M, Derksen E, Mariani C, Visser E. Rapid flooding-induced adventitious root development from preformed primordia in Solanum dulcamara. AoB PLANTS 2014;6: plt058

33. Steffens B, Rasmussen A. The Physiology of Adventitious Roots. Plant Physiology 2016;170(2):603-617

34. Yang L, Wen B. Seed Quality. Encyclopedia of Applied Plant Sciences 2017; 3:553-563

35. Shi W, Villar P, Li G, Jiang $X$. Acorn size is more important than nursery fertilization for outplanting performance of Quercus variabilis container seedlings. Annals of Forest Science 2019;76(22):12

36. Ashango T. Effect of corm and corm pieces on regeneration and multiplication of enset (Ensete ventricosum (Welw.) Cheesman). International Journal of Research - Granthaalayah 2017;5(5):281-299

37. Patiño A, Rodríguez G, Miranda T, Lemus L. Efecto de fertilización y peso del cormo sobre la multiplicación de semilla de bananito (Musa AA). Temas Agrarios 2019;24(2):139-146

38. Muhidin $M$, Nurmas $U$, Sadimantara $G$, Pratama A, Rakian T, Sutariati G, Leomo S, Yusuf D. The effect of banana humps size on the vegetative growth of dwarf banana seedling. IOP Conf. Series: Earth and Environmental Science 2021; 782:042061 
39. Rodríguez G, Ramírez $\mathrm{H}$. Efecto de diferentes sustratos y dosis de nitrógeno sobre el desarrollo de plantas de banano. In: Memoria de la XVII Reunión Internacional ACORBAT 2006 (ed), Joinville, Brasil. pp 605-615

40. Binotto A, Lúcio A, Lopes $S$. Correlations between growth variables and the Dickson quality index in forest seedlings. Cerne Lavras 2010;16(4):457-464
41. Lin K, Wu C, Chang Y. Applying Dickson Quality Index, Chlorophyll Fluorescence, and Leaf Area Index for Assessing Plant Quality of Pentas lanceolata. Not Bot Horti Agrob 2019; 47(1): 169-176

Grossnickle S, MacDonald E. Seedling quality: history, application, and plant attributes. A Review. Forests 2018;9(5):283 